\title{
A Fuzzy Logic Method for Solving Three-Tier FMCDM Problems
}

\author{
Chien-Chang Chou Kun-Ching Liao Fu-Tsuan Kuo \\ Department of Shipping Technology, National Kaohsiung Marine University, Taiwan
}

\begin{abstract}
The purpose of this paper is to propose a ranking method for solving fuzzy multiple criteria decision making (FMCDM) problems. The representation of multiplication operation on fuzzy numbers is useful for the decision makers to rank all alternatives and choose the best one under the fuzzy multiple criteria decision making environment. The representation of multiplication operation on three fuzzy numbers is proposed in this paper. Finally, this representation proposed in this paper is applied to solve the three-tier fuzzy multiple criteria decision making problem.
\end{abstract}

Keywords: Fuzzy number, Fuzzy logic, Decision making, Multiple criteria.

\section{Introduction}

The Analytic Hierarchy Process (AHP) method was first proposed by Satty. The AHP method is a popular technique often used to model subjective decision making process based on multiple criteria $[1,2]$. Many researchers have used AHP method to deal with multiple criteria decision making problems. Further, the fuzzy concept has also been introduced into the AHP method [3]. Fuzzy AHP method was used to solve fuzzy multiple attribute decision making problems $[4,5,6,7,8]$.

The concept of fuzzy sets was introduced by Zadeh [9]. The basic arithmetical operations on one fuzzy number were developed by Mizumoto and Tanaka [10], Nahmias [11], Dubois and Prade [12], $\mathrm{Ma}$ et al. [13] and Chen [14, 15]. The basic multiplication operation on two fuzzy numbers was proposed by Chou [16], and then the representation of multiplication operation on two fuzzy numbers was applied to solve the two-tier FMCDM problem: the selection of account receivable collection instrument in the international trade. The hierarchy structure for the two-tier FMCDM problem is shown in Figure 1. In many situations, the FMCDM problems are three-tier decision making problems. The hierarchy structure for the three-tier FMCDM problem is shown in Figure 2.
Thus, the purpose of this paper is to further propose the representation of multiplication operation on three fuzzy numbers, and then this representation proposed in this paper is applied to solve the three-tier FMCDM problem. Using this representation, the decision maker can rank quickly all alternatives and choose easily the best one under FMCDM environment.
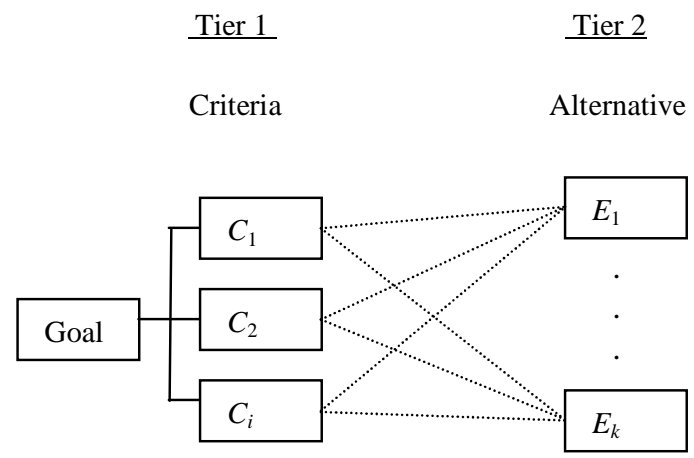

Fig. 1 The hierarchy structure for two-tier FMCDM problem.

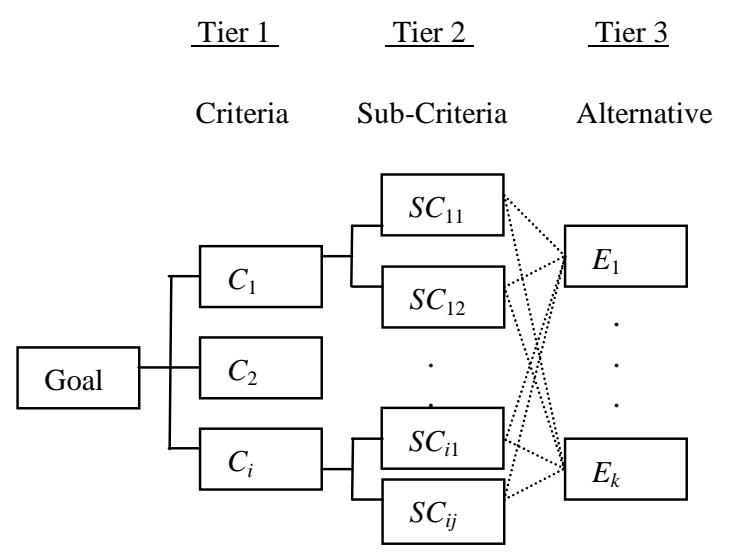

Fig. 2 The hierarchy structure for three-tier FMCDM problem.

The rest of this paper is organized as follows. The Graded Mean Integration Representation method is introduced in section 2 . In section 3 , the representation of multiplication operation on three fuzzy numbers is 
proposed. Finally, this representation of multiplication operation on three fuzzy numbers is applied to solve a numerical example of three-tier FMCDM problem in section 4 followed by conclusions in section 5 .

\section{The Graded Mean Integration Representation Method}

In 1998, Chen and Hsieh [15] proposed the graded mean integration representation method based on the integral value of graded mean $h$-level of fuzzy number. Here we describe the meaning as follows.

Let $A=(c, a, b, d)$ be a trapezoidal fuzzy number as Figure 3. Suppose the membership function of $A$ is

$$
f_{A}(x)= \begin{cases}\frac{(x-c)}{(a-c)}, & c \leq x \leq a, \\ 1, & a \leq x \leq b, \\ \frac{(x-d)}{(b-d)}, & b \leq x \leq d, \\ 0, & \text { otherwise. }\end{cases}
$$

$$
\text { Since, } \begin{aligned}
L_{A}(x)=\frac{(x-c)}{(a-c)}, & c \leq x \leq a, \\
R_{A}(x)=\frac{(x-d)}{(b-d)}, & b \leq x \leq d,
\end{aligned}
$$

and $L_{A}^{-1}(h)=c+(a-c) h, \quad 0 \leq h \leq 1$,

$$
R_{A}^{-1}(h)=d+(b-d) h, \quad 0 \leq h \leq 1,
$$

$L_{A}(x)$ and $R_{A}(x)$ are the functions $L$ and $R$ of the trapezoidal fuzzy number $A$, respectively. $L^{-1}{ }_{A}(h)$ and $R^{-1}{ }_{A}(h)$ are the inverse functions of the functions $L_{A}(x)$ and $R_{A}(x)$ at $h$-level, respectively. Then the graded mean $h$-level value of fuzzy number $A$ is $h\left[L_{A}{ }^{-1}(h)+R_{A}{ }^{-1}(h)\right] / 2$ as Figure 3 . Then the graded mean integration representation of $A$ is

$$
\begin{aligned}
& P(A)=\int_{0}^{1} \frac{h\left[L_{A}^{-1}(h)+R_{A}^{-1}(h)\right]}{2} d h / \int_{0}^{1} h d h \\
& =\int_{0}^{1} \frac{h[c+(a-c) h+d+(b-d) h]}{2} d h / \int_{0}^{1} h d h \\
& =\frac{1}{6}(c+2 a+2 b+d)
\end{aligned}
$$

Triangular fuzzy number $Y=(c, a, b)$ is a special case of trapezoidal fuzzy number. The graded mean integration representation of the triangular fuzzy number $Y$ becomes

$P(Y)=\frac{1}{6}(c+4 a+b)$

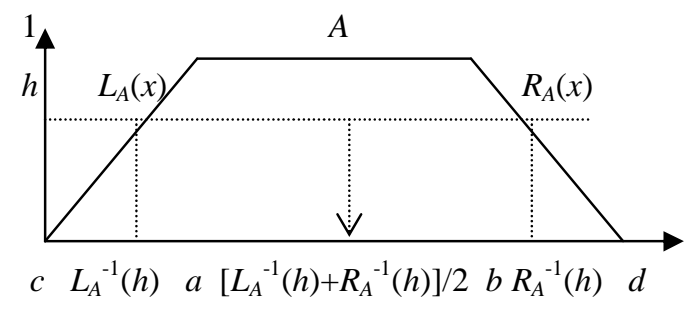

Fig. 3 The graded mean $h$-level of $A$.

\section{Representation of Multiplication on Three Fuzzy Numbers}

Based on the above-mentioned graded mean integration representation method, this paper further proposes the representation of multiplication operation on three fuzzy numbers as follows.

Definition $1 A_{1}=\left(c_{1}, a_{1}, b_{1}\right), A_{2}=\left(c_{2}, a_{2}, b_{2}\right)$ and $A_{3}=$ $\left(c_{3}, a_{3}, b_{3}\right)$ are three triangular fuzzy numbers. Let $P\left(A_{1(h)} \otimes A_{2(h)} \otimes A_{3(h)}\right)$ be the representation of $A_{1} \otimes A_{2} \otimes A_{3}$ at $h$-level. $P\left(A_{1(h)} \otimes A_{2(h)} \otimes A_{3(h)}\right)$ $=\left[L^{-1}{ }_{A 1}(h) \times L_{A 2}^{-1}(h) \times L^{-1}{ }_{A 3}(h)\right] / 8+\left[R_{A 1}^{1}(h) \times L^{-1}{ }_{A 2}(h) \times L^{-1}{ }_{A 3}(h)\right] / 8$ $+\left[L^{-1}{ }_{11}(h) \times R_{A 2}^{-1}(h) \times L^{-1}{ }_{A 3}(h)\right] / 8+\left[L^{-1}{ }_{11}(h) \times L^{-1}{ }_{A 2}(h) \times R^{-1}{ }_{A 3}(h)\right] / 8$ $+\left[L_{A 1}^{-1}(h) \times R_{A 2}^{-1}(h) \times R_{A 3}^{-1}(h)\right] / 8+\left[R_{A 1}^{1}(h) \times L_{A 2}^{-1}(h) \times R_{A 3}^{-1}(h)\right] / 8$ $+\left[R_{A 1}^{-1}(h) \times R_{A 2}^{1}(h) \times L_{A 3}^{-1}(h)\right] / 8+\left[R_{A 1}^{1}(h) \times R_{A 2}^{1}(h) \times R_{A 3}^{1}(h)\right] / 8$

$L_{A 1}(x)$ and $R_{A 1}(x)$ are the functions $L$ and $R$ of fuzzy number $\mathrm{A}_{1}$, respectively. $L^{-1}{ }_{A 1}(h)$ and $R^{-1}{ }_{A 1}(h)$ are the inverse functions of functions $L_{A 1}(x)$ and $R_{A 1}(x)$ at $h$ level, respectively. $L_{A 2}(x)$ and $R_{A 2}(x)$ are the functions $L$ and $R$ of fuzzy number $A_{2}$, respectively. $L^{-1}{ }_{A 2}(h)$ and $R^{-1}{ }_{A 2}(h)$ are the inverse functions of functions $L_{A 2}(x)$ and $R_{A 2}(x)$ at $h$-level, respectively. $L_{A 3}(x)$ and $R_{A 3}(x)$ are the functions $L$ and $R$ of fuzzy number $A_{3}$, respectively. $L^{-1}{ }_{A 3}(h)$ and $R^{-1}{ }_{A 3}(h)$ are the inverse functions of functions $L_{A 3}(x)$ and $R_{A 3}(x)$ at $h$-level, respectively.

Definition 2 Let $P\left(A_{1} \otimes A_{2} \otimes A_{3}\right)$ be the representation of $A_{1} \otimes A_{2} \otimes A_{3} . P\left(A_{1} \otimes A_{2} \otimes A_{3}\right)$

$=\int_{0}^{1} h\left\{\left[L^{-1}{ }_{A 1}(h) \times L_{A 2}^{-1}(h) \times L_{A 3}^{-1}(h)\right] / 8\right.$

$+\left[R_{A 1}^{-1}(h) \times L^{-1}{ }_{A 2}(h) \times L_{A 3}^{-1}(h)\right] / 8+\left[L_{A 1}^{-1}(h) \times R^{-1}{ }_{A 2}(h) \times L_{A 3}^{-1}(h)\right] / 8$ $+\left[L_{A 1}^{-1}(h) \times L_{A 2}^{-1}(h) \times R_{A 3}^{1}(h)\right] / 8+\left[L_{A 1}^{-1}(h) \times R_{A 2}^{-1}(h) \times R_{A 3}^{-1}(h)\right] / 8$ $+\left[R_{A 1}^{1}(h) \times L_{A 2}^{-1}(h) \times R_{A 3}^{-1}(h)\right] / 8+\left[R_{A 1}^{1}(h) \times R_{A 2}^{1}(h) \times L_{A 3}^{-1}{ }_{A 3}(h)\right] / 8$

$\left.+\left[R_{A 1}^{1}(h) \times R_{A 2}^{-1}(h) \times R_{A 3}^{-1}(h)\right] / 8\right\} d h / \int_{0}^{1} h d h$

By the above definition 2, $P\left(A_{1} \otimes A_{2} \otimes A_{3}\right)$

$=\int_{0}^{1} h\left\{\left[L^{-1}{ }_{A 1}(h) \times L_{A 2}^{-1}(h) \times L_{A 3}^{-1}(h)\right] / 8\right.$

$+\left[R_{A 1}^{-1}(h) \times L_{A 2}^{-1}(h) \times L_{A 3}^{-1}(h)\right] / 8+\left[L_{A 1}^{-1}(h) \times R_{A 2}^{-1}(h) \times L_{A 3}^{-1}(h)\right] / 8$ $+\left[L_{A 1}^{-1}(h) \times L_{A 2}^{-1}(h) \times R_{A 3}^{1}(h)\right] / 8+\left[L^{-1}{ }_{A 1}(h) \times R_{A 2}^{1}(h) \times R_{A 3}^{1}(h)\right] / 8$ $+\left[R_{A 1}^{1}(h) \times L_{A 2}^{-1}(h) \times R_{A 3}^{-1}(h)\right] / 8+\left[R_{A 1}^{1}(h) \times R_{A 2}^{1}(h) \times L_{A 3}^{-1}(h)\right] / 8$

$\left.+\left[R_{A 1}^{-1}(h) \times R_{A 2}^{-1}(h) \times R_{A 3}^{-1}(h)\right] / 8\right\} d h / \int_{0}^{1} h d h$ 
$=\frac{1}{8} \int_{0}^{1} h\left\{\left[c_{1}+\left(a_{1}-c_{1}\right) h\right] \times\left[c_{2}+\left(a_{2}-c_{2}\right) h\right] \times\left[c_{3}+\left(a_{3}-c_{3}\right) h\right]\right.$

$+\left[b_{1}+\left(a_{1}-b_{1}\right) h\right] \times\left[c_{2}+\left(a_{2}-c_{2}\right) h\right] \times\left[c_{3}+\left(a_{3}-c_{3}\right) h\right]$

$+\left[c_{1}+\left(a_{1}-c_{1}\right) h\right] \times\left[b_{2}+\left(a_{2}-b_{2}\right) h\right] \times\left[c_{3}+\left(a_{3}-c_{3}\right) h\right]$

$+\left[c_{1}+\left(a_{1}-c_{1}\right) h\right] \times\left[c_{2}+\left(a_{2}-c_{2}\right) h\right] \times\left[b_{3}+\left(a_{3}-b_{3}\right) h\right]$

$+\left[c_{1}+\left(a_{1}-c_{1}\right) h\right] \times\left[b_{2}+\left(a_{2}-b_{2}\right) h\right] \times\left[b_{3}+\left(a_{3}-b_{3}\right) h\right]$

$+\left[b_{1}+\left(a_{1}-b_{1}\right) h\right] \times\left[c_{2}+\left(a_{2}-c_{2}\right) h\right] \times\left[b_{3}+\left(a_{3}-b_{3}\right) h\right]$

$+\left[b_{1}+\left(a_{1}-b_{1}\right) h\right] \times\left[b_{2}+\left(a_{2}-b_{2}\right) h\right] \times\left[c_{3}+\left(a_{3}-c_{3}\right) h\right]$

$\left.+\left[b_{1}+\left(a_{1}-b_{1}\right) h\right] \times\left[b_{2}+\left(a_{2}-b_{2}\right) h\right] \times\left[b_{3}+\left(a_{3}-b_{3}\right) h\right]\right\} d h / \int_{0}^{1} h d h$

$=\frac{1}{8} \int_{0}^{1} h\left\{\left(c_{1} c_{2} c_{3}+b_{1} c_{2} c_{3}+c_{1} b_{2} c_{3}+c_{1} c_{2} b_{3}\right.\right.$

$\left.+c_{1} b_{2} b_{3}+b_{1} c_{2} b_{3}+b_{1} b_{2} c_{3}+b_{1} b_{2} b_{3}\right)$

$+h\left[\left(a_{3}-c_{3}\right)\left(c_{1} c_{2}+b_{1} c_{2}+c_{1} b_{2}+b_{1} b_{2}\right)\right.$

$+\left(a_{2}-c_{2}\right)\left(c_{1} c_{3}+b_{1} c_{3}+c_{1} b_{3}+b_{1} b_{3}\right)$

$+\left(a_{1}-c_{1}\right)\left(c_{2} c_{3}+b_{2} c_{3}+c_{2} b_{3}+b_{2} b_{3}\right)$

$+\left(a_{3}-b_{3}\right)\left(c_{1} c_{2}+b_{1} c_{2}+c_{1} b_{2}+b_{1} b_{2}\right)$

$+\left(a_{2}-b_{2}\right)\left(c_{1} c_{3}+b_{1} c_{3}+c_{1} b_{3}+b_{1} b_{3}\right)$

$\left.+\left(a_{1}-b_{1}\right)\left(c_{2} c_{3}+b_{2} c_{3}+c_{2} b_{3}+b_{2} b_{3}\right)\right]$

$+h^{2}\left[\left(a_{2}-c_{2}\right)\left(a_{3}-c_{3}\right)\left(b_{1}+c_{1}\right)+\left(a_{2}-b_{2}\right)\left(a_{3}-c_{3}\right)\left(b_{1}+c_{1}\right)\right.$

$+\left(a_{2}-c_{2}\right)\left(a_{3}-b_{3}\right)\left(b_{1}+c_{1}\right)+\left(a_{2}-b_{2}\right)\left(a_{3}-b_{3}\right)\left(b_{1}+c_{1}\right)$

$+\left(a_{1}-c_{1}\right)\left(a_{3}-c_{3}\right)\left(b_{2}+c_{2}\right)+\left(a_{1}-b_{1}\right)\left(a_{3}-c_{3}\right)\left(b_{2}+c_{2}\right)$

$+\left(a_{1}-c_{1}\right)\left(a_{3}-b_{3}\right)\left(b_{2}+c_{2}\right)+\left(a_{1}-b_{1}\right)\left(a_{3}-b_{3}\right)\left(b_{2}+c_{2}\right)$

$+\left(a_{1}-c_{1}\right)\left(a_{2}-c_{2}\right)\left(b_{3}+c_{3}\right)+\left(a_{1}-b_{1}\right)\left(a_{2}-c_{2}\right)\left(b_{3}+c_{3}\right)$

$\left.+\left(a_{1}-c_{1}\right)\left(a_{2}-b_{2}\right)\left(b_{3}+c_{3}\right)+\left(a_{1}-b_{1}\right)\left(a_{2}-b_{2}\right)\left(b_{3}+c_{3}\right)\right]$

$+h^{3}\left[\left(a_{1}-b_{1}\right)\left(a_{2}-c_{2}\right)\left(a_{3}-c_{3}+a_{3}-b_{3}\right)\right.$

$+\left(a_{1}-c_{1}\right)\left(a_{2}-c_{2}\right)\left(a_{3}-c_{3}+a_{3}-b_{3}\right)+\left(a_{1}-c_{1}\right)\left(a_{2}-b_{2}\right)\left(a_{3}-c_{3}+a_{3}-b_{3}\right)$

$\left.\left.+\left(a_{1}-b_{1}\right)\left(a_{2}-b_{2}\right)\left(a_{3}-c_{3}+a_{3}-b_{3}\right)\right]\right\} d h / \int_{0}^{1} h d h$

$=\frac{1}{8} \int_{0}^{1} h\left\{\left(c_{1} c_{2}\left(c_{3}+b_{3}\right)+b_{1} c_{2}\left(c_{3}+b_{3}\right)\right.\right.$

$+c_{1} b_{2}\left(c_{3}+b_{3}\right)+b_{1} b_{2}\left(c_{3}+b_{3}\right)$

$+h\left[\left(a_{3}-c_{3}+a_{3}-b_{3}\right)\left(c_{1} c_{2}+b_{1} c_{2}+c_{1} b_{2}+b_{1} b_{2}\right)\right.$

$+\left(a_{2}-c_{2}+a_{2}-b_{2}\right)\left(c_{1} c_{3}+b_{1} c_{3}+c_{1} b_{3}+b_{1} b_{3}\right)$

$+\left(a_{1}-c_{1}+a_{1}-b_{1}\right)\left(c_{2} c_{3}+b_{2} c_{3}+c_{2} b_{3}+b_{2} b_{3}\right)$

$+h^{2}\left[\left(a_{3}-c_{3}\right)\left(b_{1}+c_{1}\right)\left(a_{2}-c_{2}+a_{2}-b_{2}\right)\right.$

$+\left(a_{3}-b_{3}\right)\left(b_{1}+c_{1}\right)\left(a_{2}-c_{2}+a_{2}-b_{2}\right)$

$+\left(a_{3}-c_{3}\right)\left(b_{2}+c_{2}\right)\left(a_{1}-c_{1}+a_{1}-b_{1}\right)+\left(a_{3}-b_{3}\right)\left(b_{2}+c_{2}\right)\left(a_{1}-c_{1}+a_{1}-b_{1}\right)$

$\left.+\left(a_{2}-c_{2}\right)\left(b_{3}+c_{3}\right)\left(a_{1}-c_{1}+a_{1}-b_{1}\right)+\left(a_{2}-b_{2}\right)\left(b_{3}+c_{3}\right)\left(a_{1}-c_{1}+a_{1}-b_{1}\right)\right]$

$+h^{3}\left[\left(a_{2}-c_{2}\right)\left(a_{3}-c_{3}+a_{3}-b_{3}\right)\left(a_{1}-b_{1}+a_{1}-c_{1}\right)\right.$

$\left.\left.+\left(a_{2}-b_{2}\right)\left(a_{3}-c_{3}+a_{3}-b_{3}\right)\left(a_{1}-c_{1}+a_{1}-b_{1}\right)\right]\right\} d h / \int_{0}^{1} h d h$

$=\frac{1}{8} \int_{0}^{1} h\left\{\left(c_{3}+b_{3}\right)\left(c_{2}+b_{2}\right)\left(c_{1}+b_{1}\right)+h\left[\left(a_{3}-c_{3}+a_{3}-b_{3}\right)\left(c_{2}+b_{2}\right)\left(c_{1}+b_{1}\right)\right.\right.$

$\left.+\left(a_{2}-c_{2}+a_{2}-b_{2}\right)\left(c_{3}+b_{3}\right)\left(c_{1}+b_{1}\right)+\left(a_{1}-c_{1}+a_{1}-b_{1}\right)\left(c_{3}+b_{3}\right)\left(c_{2}+b_{2}\right)\right]$

$+h^{2}\left[\left(a_{3}-c_{3}+a_{3}-b_{3}\right)\left(b_{1}+c_{1}\right)\left(a_{2}-c_{2}+a_{2}-b_{2}\right)\right.$

$+\left(a_{3}-c_{3}+a_{3}-b_{3}\right)\left(b_{2}+c_{2}\right)\left(a_{1}-c_{1}+a_{1}-b_{1}\right)$

$\left.+\left(a_{2}-c_{2}+a_{2}-b_{2}\right)\left(b_{3}+c_{3}\right)\left(a_{1}-c_{1}+a_{1}-b_{1}\right)\right]$

$\left.+h^{3}\left[\left(a_{2}-c_{2}+a_{2}-b_{2}\right)\left(a_{3}-c_{3}+a_{3}-b_{3}\right)\left(a_{1}-c_{1}+a_{1}-b_{1}\right)\right]\right\} d h / \int_{0}^{1} h d h$

$=\frac{1}{8}\left(c_{3}+b_{3}\right)\left(c_{2}+b_{2}\right)\left(c_{1}+b_{1}\right)+\frac{1}{12}\left[\left(a_{3}-c_{3}+a_{3}-b_{3}\right)\left(c_{2}+b_{2}\right)\left(c_{1}+b_{1}\right)\right.$

$\left.+\left(a_{2}-c_{2}+a_{2}-b_{2}\right)\left(c_{3}+b_{3}\right)\left(c_{1}+b_{1}\right)+\left(a_{1}-c_{1}+a_{1}-b_{1}\right)\left(c_{3}+b_{3}\right)\left(c_{2}+b_{2}\right)\right]$ $+\frac{1}{16}\left[\left(a_{3}-c_{3}+a_{3}-b_{3}\right)\left(b_{1}+c_{1}\right)\left(a_{2}-c_{2}+a_{2}-b_{2}\right)+\left(a_{3}-c_{3}+a_{3}-b_{3}\right)\right.$

$\left.\left(b_{2}+c_{2}\right)\left(a_{1}-c_{1}+a_{1}-b_{1}\right)+\left(a_{2}-c_{2}+a_{2}-b_{2}\right)\left(b_{3}+c_{3}\right)\left(a_{1}-c_{1}+a_{1}-b_{1}\right)\right]$

$+\frac{1}{20}\left[\left(a_{2}-c_{2}+a_{2}-b_{2}\right)\left(a_{3}-c_{3}+a_{3}-b_{3}\right)\left(a_{1}-c_{1}+a_{1}-b_{1}\right)\right]$

We have that $P\left(A_{1} \otimes A_{2} \otimes A_{3}\right)$

$=\frac{1}{8}\left(c_{1}+b_{1}\right)\left(c_{2}+b_{2}\right)\left(c_{3}+b_{3}\right)+\frac{1}{12}\left[\left(c_{1}+b_{1}\right)\left(c_{2}+b_{2}\right)\left(a_{3}-c_{3}+a_{3}-b_{3}\right)\right.$

$\left.+\left(c_{1}+b_{1}\right)\left(a_{2}-c_{2}+a_{2}-b_{2}\right)\left(c_{3}+b_{3}\right)+\left(a_{1}-c_{1}+a_{1}-b_{1}\right)\left(c_{2}+b_{2}\right)\left(c_{3}+b_{3}\right)\right]$

$+\frac{1}{16}\left[\left(b_{1}+c_{1}\right)\left(a_{2}-c_{2}+a_{2}-b_{2}\right)\left(a_{3}-c_{3}+a_{3}-b_{3}\right)+\left(a_{1}-c_{1}+a_{1}-b_{1}\right)\right.$

$\left.\left(b_{2}+c_{2}\right)\left(a_{3}-c_{3}+a_{3}-b_{3}\right)+\left(a_{1}-c_{1}+a_{1}-b_{1}\right)\left(a_{2}-c_{2}+a_{2}-b_{2}\right)\left(b_{3}+c_{3}\right)\right]$

$+\frac{1}{20}\left[\left(a_{1}-c_{1}+a_{1}-b_{1}\right)\left(a_{2}-c_{2}+a_{2}-b_{2}\right)\left(a_{3}-c_{3}+a_{3}-b_{3}\right)\right]$

For example, let $A_{1}=\left(c_{1}, a_{1}, b_{1}\right)=(0,1,2), A_{2}=\left(c_{2}, a_{2}, b_{2}\right)$ $=(1,2,3)$ and $A_{3}=\left(c_{3}, a_{3}, b_{3}\right)=(2,3,4)$ be three triangular fuzzy numbers. The representation of $A_{1}$ is $P\left(A_{1}\right)=1$. The representation of $A_{2}$ is $P\left(A_{2}\right)=2$. The representation of $A_{3}$ is $P\left(A_{3}\right)=3$. $P\left(A_{1}\right) \times P\left(A_{2}\right) \times P\left(A_{3}\right)$ $=1 \times 2 \times 3=6$.

By formula (3) proposed in this paper, the representation of $A_{1} \otimes A_{2} \otimes A_{3}$ is $P\left(A_{1} \otimes A_{2} \otimes A_{3}\right)=6$. We have that

$P\left(A_{1}\right) \otimes P\left(A_{2}\right) \otimes P\left(A_{3}\right)=6=P\left(A_{1} \otimes A_{2} \otimes A_{3}\right)$

\section{A Example of Three-Tier FMCDM}

The representation of multiplication operation on three fuzzy numbers proposed in this paper is applied to solve the following numerical example of three-tier FMCDM in Figure 4. The basic data is shown as follows.

Alternatives: $E_{1}$ and $E_{2}$

Criteria: $C_{1}$ and $C_{2}$

Sub-Criteria: $S C_{11}, S C_{12}, S C_{21}$ and $S C_{22}$

Importance weight of $C_{1}: W_{c 1}=(0.2,0.3,0.4)$

Importance weight of $C_{2}: W_{c 2}=(0.6,0.7,0.8)$

Importance weight of $S C_{11}: W_{s c 11}=(0.5,0.6,0.7)$

Importance weight of $S C_{12}: W_{s c 12}=(0.3,0.4,0.5)$

Importance weight of $S C_{21}: W_{s c 21}=(0.5,0.6,0.7)$

Importance weight of $S C_{22}: W_{s c 22}=(0.3,0.4,0.5)$

Preference of $E_{1}$ under $S C_{11}: P_{E I S C 11}=(0.7,0.8,0.9)$

Preference of $E_{1}$ under $S C_{12}: P_{E I S C 12}=(0.8,0.9,1.0)$

Preference of $E_{1}$ under $S C_{21}: P_{E I S C 21}=(0.5,0.6,0.7)$

Preference of $E_{1}$ under $S C_{22}: P_{E I S C 22}=(0.6,0.7,0.8)$

Preference of $E_{2}$ under $S C_{11}: P_{E 2 S C 11}=(0.5,0.6,0.7)$

Preference of $E_{2}$ under $S C_{12}: P_{E 2 S C 12}=(0.6,0.7,0.8)$

Preference of $E_{2}$ under $S C_{21}: P_{E 2 S C 21}=(0.7,0.8,0.9)$

Preference of $E_{2}$ under $S C_{22}: P_{E 2 S C 22}=(0.8,0.9,1.0)$ 


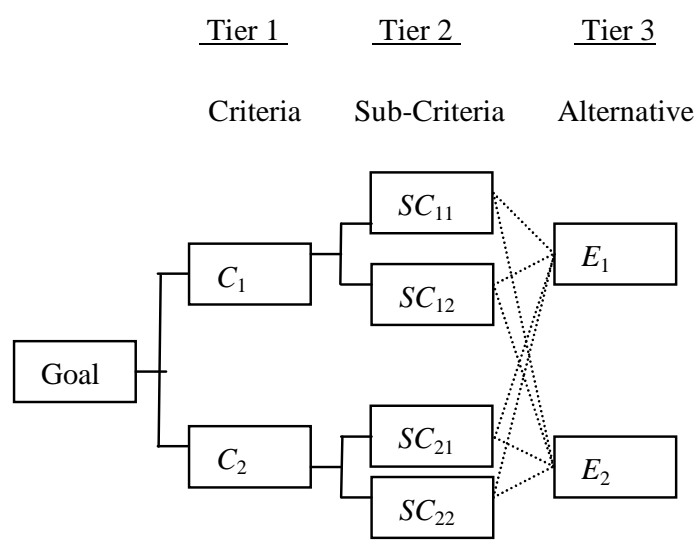

Fig. 4 The hierarchy structure for three-tier FMCDM problem.

Let the total performance of alternative $\mathrm{E}_{1}$ is $P_{E 1}$. Let the total performance of alternative $\mathrm{E}_{2}$ is $P_{E 2}$. $P_{E 1}=P_{E 1 S C 11} \otimes W_{S C 11} \otimes W_{C 1}+P_{E 1 S C 12} \otimes W_{S C 12} \otimes W_{C 1}$ $+P_{E 1 S C 21} \otimes W_{S C 21} \otimes W_{C 2}+P_{E 1 S C 22} \otimes W_{S C 22} \otimes W_{C 2}$ $=(0.7,0.8,0.9) \otimes(0.5,0.6,0.7) \otimes(0.2,0.3,0.4)$ $+(0.8,0.9,1.0) \otimes(0.3,0.4,0.5) \otimes(0.2,0.3,0.4)$ $+(0.5,0.6,0.7) \otimes(0.5,0.6,0.7) \otimes(0.6,0.7,0.8)$ $+(0.6,0.7,0.8) \otimes(0.3,0.4,0.5) \otimes(0.6,0.7,0.8)$

$P_{E 2}=P_{E 2 S C 11} \otimes W_{S C 11} \otimes W_{C 1}+P_{E 2 S C 12} \otimes W_{S C 12} \otimes W_{C 1}$ $+P_{E 2 S C 21} \otimes W_{S C 21} \otimes W_{C 2}+P_{E 2 S C 22} \otimes W_{S C 22} \otimes W_{C 2}$ $=(0.5,0.6,0.7) \otimes(0.5,0.6,0.7) \otimes(0.2,0.3,0.4)$ $+(0.6,0.7,0.8) \otimes(0.3,0.4,0.5) \otimes(0.2,0.3,0.4)$ $+(0.7,0.8,0.9) \otimes(0.5,0.6,0.7) \otimes(0.6,0.7,0.8)$ $+(0.8,0.9,1.0) \otimes(0.3,0.4,0.5) \otimes(0.6,0.7,0.8)$

By formula (3), $P_{E 1}=0.7, P_{E 2}=0.78$. We have that $P_{E 1}<$ $P_{E 2}$. The alternative $E_{2}$ is selected.

\section{Conclusion}

This paper proposed the representation of multiplication operation on three fuzzy numbers. Finally, this representation is applied to solve a numerical example of three-tier FMCDM problem. By this representation, the decision maker can rank quickly all alternatives and select the best one under FMCDM environment. The representation proposed in this paper will be applied to empirical studies on threetiers FMCDM problems in the future studies.

\section{References}

[1] T. L. Saaty, "A scaling method for priorities in hierarchical structure", Journal of Mathematical Psychology, Vol. 15(2), pp. 274-281, 1977.

[2] T. L. Saaty, "The Analytic Hierarchy Process", McGraw-Hill Inc., New York, 1980.

[3] J. J. Buckley, "Ranking alternatives using fuzzy numbers", Fuzzy Sets and Systems, Vol. 15(1), pp. 21-31, 1985.

[4] H. K. Chiou, and G. H. Tzeng, "Fuzzy multiple criteria decision making approach for industrial green engineering", Environment Management, Vol. 30(6), pp. 816-830, 2002.

[5] T. Y. Chou, and G. S. Liang, "Application of a fuzzy multiple-criteria decision-making model for shipping performance evaluation", Maritime Policy and Management, Vol. 28(4), pp. 375-392, 2001

[6] M. T. Tang, G. H. Tzeng, and S. W. Wang, "A hierarchy fuzzy MCDM method for studying electronic marketing strategies in the information service industry", Journal of International Information Management, Vol. 8(1), pp. 1-22, 1999.

[7] J. Y. Teng, and G. H. Tzeng, "Fuzzy multiplecriteria ranking of urban transportation investment alternative", Transportation Planning and Technology, Vol. 20(1), pp. 15-31, 1996.

[8] S. H. Tsaur, T. Y. Chang, and C. H. Yen, "The evaluation of airline service quality by fuzzy MCDM", Tourism Management, Vol. 23, pp. 107-115, 2002.

[9] L. A. Zadeh, "The concept of a linguistic variable and its application to approximate reasoning", Information Sciences, Vol. 8, pp. 199-249(1), pp. 301-357(2), 1975.

[10] M. Mizumoto, and K. Tanaka, "The four operations of arithmetic on fuzzy numbers", Systems Comput. Controls 7(5), pp. 73-81, 1976.

[11] S. Nahmias, "Fuzzy variables", Fuzzy Sets and Systems, pp. 97-111, 1978.

[12] D. Dubois, and H. Prade, "Operations on fuzzy numbers", Journal of Systems Sciences Vol. 9, pp. 613-626, 1978.

[13] M. Ma, M. Friedman, A. Kandel, "A new fuzzy arithmetic", Fuzzy Sets and Systems Vol. 108, pp. 83-90, 1999.

[14] S. H. Chen, "Operations of fuzzy numbers with step form membership function using function principle”, Information Sciences Vol. 108, pp. 149155, 1998.

[15] S. H. Chen, and C. H. Hsieh, "Graded mean integration representation of generalized fuzzy number", Proceeding of 1998 sixth Conference on Fuzzy Theory and its Application (CD-ROM), filename: 031.wdl, Chinese Fuzzy Systems Association, Taiwan, Republic of China, pp. 1-6, 1998.

[16] C. C. Chou, "A fuzzy multiple criteria decision making method", Proceeding of $2^{\text {nd }}$ IEEE International Conference on Cybernetics \& Intelligent Systems, pp. 723-726, 2006. 